\title{
Optimal Radius for Connectivity in Duty-Cycled Wireless Sensor Networks
}

\author{
Amitabha Bagchi \\ Dept. of Comp. Sci. and Engg. \\ IIT Delhi, India \\ bagchi@cse.iitd.ernet.in
}

\author{
Cristina M. Pinotti \\ Dept. of Comp. Sci. and Math. \\ University of Perugia, Italy \\ cristina.pinotti@unipg.it \\ Tarun Mangla \\ Dept. of Comp. Sci. and Engg. \\ IIT Delhi, India \\ cs1100260@cse.iitd.ernet.in
}

Sainyam Galhotra

Dept. of Comp. Sci. and Engg.

IIT Delhi, India

cs1100246@cse.iitd.ernet.in

\begin{abstract}
We investigate the condition on transmission radius needed to achieve connectivity in duty-cycled wireless sensor networks (briefly, DC-WSN). First, we settle a conjecture of Das et. al. (2012) and prove that the connectivity condition on Random Geometric Graphs (RGG), given by Gupta and Kumar (1989), can be used to derive a weak sufficient condition to achieve connectivity in DC-WSN. We also present a stronger result which gives a necessary and sufficient condition for connectivity and is hence optimal. The optimality of such a radius is also tested via simulation for two specific duty-cycle schemes, called the contiguous and the random selection duty-cycle scheme.
\end{abstract}

\section{Categories and Subject Descriptors}

C.2.1 [Network Architecture and Design]: Wireless Communication

\section{General Terms}

Theory, Design, Performance, Experimentation

\section{Keywords}

duty-cycled wireless sensor networks; connectivity radius

\section{INTRODUCTION}

Careful control of transmission power is required in wireless sensor networks to ensure that data can be sent while wasting minimum energy in interference and collision. This tradeoff translates into the question: what is the optimal radius for the connectivity of the WSN graph? This has been

\footnotetext{
${ }^{*}$ This work has been partially supported by the Research Grant 2010N5K7EB from MIUR and Project Sapienza 2011 "Green and Secure Wireless Networking".
}

Permission to make digital or hard copies of all or part of this work for personal or classroom use is granted without fee provided that copies are not made or distributed for profit or commercial advantage and that copies bear this notice and the full citation on the first page. Copyrights for components of this work owned by others than ACM must be honored. Abstracting with credit is permitted. To copy otherwise, or republish, to post on servers or to redistribute to lists, requires prior specific permission and/or a fee. Request permissions from permissions@acm.org.

MSWiM'13, November 3-8, 2013, Barcelona, Spain.

Copyright 2013 ACM 978-1-4503-2353-6/13/11

http://dx.doi.org/10.1145/2507924.2507985 ...\$15.00. studied using the Random Geometric Graph (RGG) model by Gupta and Kumar [3] among others.

To save energy even during sensing and processing activities, often sensors are organized in Duty-Cycled Wireless Sensor Networks (briefly, DC-WSNs). In such networks, sensors alternate between the awake and the sleep mode. During the sleep mode, the sensors recharge or conserve their batteries; during the active mode, the sensors sense, process and communicate. However, for the duty-cycled network to function as it should, we need two properties: (a) Time coverage, i.e. data generated at any time must be sensed and relayed by the network, and (b) connectivity, i.e. every node should be connected to every other node. The study of the conditions that guarantee these properties is the focus of this paper. This problem was initially investigated in [2], where it was conjectured that the DC-WSN is connected if in every time slot the nodes awake form a RGG connected in that time slot. We have settled this conjecture although we do not have space to present the proof here [1]. We have also established that the radius of connectivity that this conjecture implies is not optimal, i.e. a lower connection radius is sufficient. This lower connection radius, we call it the strong radius as opposed to the weak radius conjectured in [2], is also to be optimal in the sense that it provides a necessary condition for connectivity. We present two natural duty-cycling schemes, called the contiguous scheme and the random selection scheme that both satisfy the time coverage property. Apart from being useful duty-cycling schemes for real applications, these schemes also highlight the contribution of this paper since they have the same weak radius of connectivity but very different strong radii.

\section{Related work and Motivation.}

Power conservation mechanisms for WSNs may be classified into two main categories: active and passive [4]. Active mechanisms achieve energy conservation by smartly defining energy-efficient network protocols. Since they do not turn off the sensors, the connectivity property of the wireless sensor networks is preserved. Passive mechanisms save power by turning off the radio/sensing sensor module. As surveyed in [4], passive mechanisms can apply turn-off techniques at the physical layer for reducing the CPU energy in idle system states, or at the MAC layer by leading such a layer to decide when there is a frame destinated to it and then turn- 
off the radio interface for the remaining time of the frame, or utilizing higher layer application informations to decide when turn-off the radio. Since turning off the sensors makes the topology of the wireless sensor networks dynamic, the connectivity of such networks needs to be investigated.

\section{THE DUTY-CYCLE MODEL}

We first define our notation and the model following [2]. A random geometric graph $\mathrm{RGG}(n, r)$ is a graph with vertex set $V$ of $n$ points distributed uniformly at random in the unit circle centred at the origin. To this we add a point at the origin. There are edges between any two $u, v \in V$ such that $d(u, v) \leq r$ where $d(\cdot, \cdot)$ is a distance defined on $\mathbb{R}^{2}$.

In the duty-cycled setting all the nodes in the network are assumed to be synchronized and following a periodic pattern of sleeping and waking. Although the sleeping and waking patterns of different sensors may be different, they all share the same period, and each cycle of this periodic pattern (known as a duty cycle) begins and ends at the same time for all the nodes. As described in [2], the primary parameters of a duty-cycled wireless sensor network are L, length of the duty cycle, and $d$, the number of waking slots where $d<L$ and we use the notation $\delta=\lceil d / L\rceil$ to indicate the $d u t y$ cycle ratio, which is a measure of the energy spent by each sensor in each cycle. Each sensor $u$ chooses its waking slots which we denote by the set $A_{u}$ where $A_{u} \subseteq\{0,1, \ldots, L-1\}$ and $\left|A_{u}\right|=d$. Given a scheme $\mathcal{A}$ for choosing these waking slots, we define the duty-cycle graph $\operatorname{DC}_{-W_{\mathcal{A}}}(n, r, \delta, L)$ as follows: it has the same vertex set as $\operatorname{RGG}(n, r)$ and its edge set is: $E=\left\{(u, v): d(u, v) \leq r, A_{u} \cap A_{v} \neq \emptyset\right\}$. Namely, for two vertices $u$ and $v$ that are within transmission range of each other to be connected, they must share a slot where they are both awake. When the context requires it, we will also use the notation $\mathrm{DC}_{-\mathrm{WSN}_{\mathcal{A}}}(n, r, \delta, L, \gamma)$ to denote a duty-cycle graph wherein the probability of two vertices $u$ and $v$ that are within transmission range of each other sharing a waking slot is $\gamma$.

A fundamental property desired of any duty-cycled sensor network is connectivity i.e. it should be possible to send data generated at any time at any node to any other node in the network (within reasonable time).

Whenever the awake period of each sensor is (strictly) more than half the duty cycle then each edge of the original graph $\operatorname{RGG}(n, r)$ is available for at least one time slot because any two waking periods must share a slot, i.e.

FACT 2.1. If $\delta>1 / 2$ then $D C-W S N(n, r, \delta, L)$ is connected whenever $R G G(n, r)$ is connected.

However, for $\delta \leq 1 / 2$, connectivity is not a given under our current definition. Not only is it a random event whose probability needs to be determined, it may also be an event which occurs with probability 0 . To ensure that the model admits connectivity with non-zero probability we will need a technical condition we call the reachability condition. Consider a scheme $\mathcal{A}$ for selecting the waking slots of nodes. Given the set $\mathcal{L}=\{A: A \subset\{0,1, \ldots, L-1\},|A|=d\}$, let us denote by $\mathcal{L}(\mathcal{A})$ all those subsets in $\mathcal{L}$ that have non-zero probability of being selected as a waking schedule for a node. Then, the reachability condition on $\mathcal{A}$ is the following:

Reachability. There is a finite $k \geq 0$ such that for any $A_{1}, A_{2} \in \mathcal{L}(\mathcal{A})$, there are $A_{1}=B_{0}, \ldots, B_{k}=A_{2}$ such that $B_{i} \in \mathcal{L}(\mathcal{A}), 0 \leq i \leq k$, and $B_{i} \cap B_{i+1} \neq \emptyset, 0 \leq i<k$.
We also need a notion which ensures that a duty-cycling scheme does not allow time slots where no sensor is awake. We call this notion time coverage.

Time coverage. For each $k \in\{0,1, \ldots, L-1\}$, the probability that a node $u$ is awake in slot $k$ is $\delta_{k}>0$, where $\delta_{k}$ may be a function of $d$ and $L$ but is not dependent on the number of nodes in the network.

If all the $\delta_{k}$ are equal then we say that there is uniform coverage.

\section{CONNECTIVITY RESULTS}

In this section we present a weak and a strong condition on transmission radius needed to achieve connectivity.

\section{The weak result.}

The weak result Theorem 3.1 is a generalization of the result first presented in Das et. al. [2].

Theorem 3.1. Given a duty-cycling scheme $\mathcal{A}$ with $0<$ $\delta \leq 1 / 2$ and $d=\lceil\delta L\rceil>1$, and the marginal probability of a node being awake in slot $i$ denoted by $\delta_{i}$, the probability that $D C-W S N_{\mathcal{A}}(n, r(n), \delta, L)$ is connected tends to 1 as $n \rightarrow$ $\infty$ if $\mathcal{A}$ satisfies the reachability condition and the coverage condition and if

$$
\pi r^{2}(n) \delta_{\min }=(\log n+c(n)) / n,
$$

such that $c(n) \rightarrow \infty$ as $n \rightarrow \infty$, where $\delta_{\min }=\min _{k=0}^{L-1} \delta_{k}$.

We prove the theorem by considering a set of $L$ subgraphs of $\mathrm{DC}_{-\mathrm{WSN}_{\mathcal{A}}}(n, r, \delta, L)$, one for each time slot in a typical duty cycle. The proof proceeds by using Gupta and Kumar's result to show that each of these subgraphs is connected with probability that goes to 1 as $n \rightarrow \infty$ and that these subgraphs are all connected with probability tending to 1 as $n \rightarrow \infty$. We omit details of the proof due to lack of space.

In the uniform coverage situation, i.e. $\delta_{0}=\ldots=\delta_{L-1}=$ $\delta=\delta_{\min }$, Theorem 3.1 gives us the theorem of [2].

\section{The strong result.}

In this section we develop and present our strong connectivity result for duty-cycled WSNs, i.e. the most important contribution of our paper. Proving this result involves defining a new "vertex-based" random connection model. This model has four parameters. There are two finite positive real numbers $\lambda, r$. The third parameter is a random variable $Z$ defined on some probability space $(\Omega, \mathcal{F}, \mathrm{P})$, that is a function of the form $Z: \Omega \rightarrow Q$ where $Q$ is some domain. The fourth parameter is a function $f: Q \times Q \rightarrow\{0,1\}$. The vertex set $V$ is a Poisson point process in $\mathbb{R}^{2}$ with density $\lambda$ with an additional point at the origin. With each $u \in V$ we associate a random variable $Z_{u}$ which is a copy of $Z$. All the random variables in the collection $\left\{Z_{u}: u \in V\right\}$ are independent of each other. Two vertices $u$ and $v$ with distance $r$ are connected by an edge if $f\left(Z_{u}, Z_{v}\right)=1$. Clearly, for this model to be useful, there should be non-zero probability of an edge being formed between two points that are within distance $r$ of each other.

Since the vertex-based random connection model is a new continuum percolation model, it is necessary to establish certain properties like the existence of a critical density for percolation (i.e. the formation of an infinite component), the non-triviality of the critical density, and the uniqueness of the infinite component. Since this is not directly relevant 
to the duty-cycled networks setting and due to the lack of space, we omit it here.

We now come to our main result dealing with the highdensity situation. Define the quantity $q_{k}(\lambda)=\mathrm{P}_{\lambda}(|W|=$ $k), k \geq 1$ where $W$ is the connected component containing the origin i.e. $q_{k}(\lambda)$ is the probability that the component containing the origin has size $k$ for all finite values of $k$. Our key contribution is that we can prove that the following result proved by Penrose [5] for the high-density setting of the random connection model also holds for the vertex-based random connection model:

LEMma 3.2.

$$
\lim _{\lambda \rightarrow \infty} \frac{\sum_{k=1}^{\infty} q_{k}(\lambda)}{q_{1}(\lambda)}=1
$$

Since $\sum_{k=1}^{\infty} q_{k}(\lambda)$ is the probability that the origin is part of a finite sized component with at least 2 points in it, the implication of the theorem is that as $\lambda \rightarrow \infty$, the origin is either isolated or part of the infinite component with probability 1.

We are now ready to denote by $\operatorname{VB}-\operatorname{RGG}(n, r, \gamma)$, the vertex-based random connection model graph with vertex set consisting of $n$ points uniformly distributed in the unit circle centred at the origin, with radius bound $r$, and a connectivity function $g$ that uses a function $f$ and and random variable $Z$ such that for any $Z_{1}$ and $Z_{2}$ that are independent copies of $Z, \gamma=\mathrm{P}\left(f\left(Z_{1}, Z_{2}\right)=1\right)$. Now, we are ready to state our strong connectivity result in Theorem 3.3. We omit the proof for space constraints.

Theorem 3.3. $P\left(D C-W S N_{\mathcal{A}}(n, r, \delta, L, \gamma)\right.$ is connected $) \rightarrow$ 1 as $n \rightarrow \infty$ if and only if

$$
\pi r(n)^{2} \gamma=(\log n+c(n)) / n
$$

where $c=\lim _{n \rightarrow \infty} c(n)=\infty$ as $n \rightarrow \infty$.

\section{SIMULATION RESULTS}

In this section, we illustrate the significance of our results for two specific duty-cycle schemes: the contiguous (we will denote it DC-C-WSN) and the random selection (we will denote it DC-R-WSN) schemes. The contiguous model has been studied in [2]. There $A_{u}$ is chosen as follows: Each sensor $u$ independently chooses an integer $i_{u}$ from the set $\{0,1, \ldots, L-1\}$ and is awake for the all the time slots $i_{u}$ to $i_{u}+d-1$, then asleep from all the time slots $i_{u}+d$ to $i_{u}+L$, repeating this cycle indefinitely. In the random selection model, each node chooses a set $A_{u}$ of size $d$ at random from $\{0,1, \ldots, L-1\}$.

In the rest of this section, we first test the weak radius by measuring the percentage of sensors belonging to the largest component in DC-C-WSN and DC-R-WSN for various values of $n, L$ and $\delta$. Then, we repeat the experiments using the optimal radius.

\section{Experimental setup.}

In regard to the duty-cycle parameters, we select $\delta$ varying between 0.05 and 0.5 according to Fact 2.1. Then either we calculate $d=\lceil\delta L\rceil$ by fixing $L=100$, or we derive $L=\lceil d / \delta\rceil$ by fixing $d=5$. In addition to $d, L$, and $r(n)$, some informations are stored in the file for each sensor, depending on the duty-cycle scheme. For DC-C-WSN, we store for each sensor its start time, generated at random. For DC-R-WSN, we store a bitmap of length $L$. This bitmap will have $d$ of its bits set to 1 , which implies that the sensor is awake in that time slot, and the rest of them set to 0 . To add an edge between two sensors, we check if they lie within the connectivity radius and if they share a common awake slot. To find the connected component, we make use of the Union Find algorithm. We start assigning unique flags to each point (i.e., each sensor belongs to an isolated component). Initially these flags point to themselves. As and when we get an edge between two points, we combine the connected components of both the points by pointing the head flag of one component to the head flag of the other component. When all edges have been added, the number of the distinct connected components in the graph and their sizes are traced. Each experiment is repeated at least three times and the average value is reported. The $k d$-tree spatial data structure was used to process pairs of points in $\theta(n)$ time, and the connected components were created in $\theta(n \log n)$ time. As a result testing connectivity for a random graph model with $10^{6}$ node took approximately 20 minutes.

\section{Weak connectivity condition.}

Let the weak radius be the radius $r(n)$ that satisfies Theorem 3.1. Since both contiguous and random selection schemes satisfies the uniform coverage situation with $\delta=\lceil d / L\rceil$, the weak radius for both schemes yields:

$$
r(n)=\sqrt{\frac{\log n+c(n)}{\pi n \delta}}
$$

with $c(n) \rightarrow+\infty$ as $n \rightarrow+\infty$.

Under the weak radius, in all our experiments in DC-C-WSNs more than $99 \%$ sensors belong to the largest connected component, while all the sensors belong to the largest component in DC-R-WSNs. We omit these curves for lack of space.

Note that the weak radius is much larger than the transmission radius required to achieve the connectivity in RGG. In fact it is $\frac{1}{\sqrt{\delta}}$ times the $\mathrm{RGG}$ radius given in [3]. Such an increase in the transmission radius means on average that, since the power spent by each node in transmission is proportional to the square of the radius and since $n \delta$ sensors are awake, the overall energy spent is almost the same as in regular (i.e. all awake) WSNs, thereby negating the effect of duty-cycling.

\section{Strong connectivity condition.}

To apply the strong connectivity condition in Theorem 3.3 for the contiguous and random duty-cycle scheme, we need to compute the probability $\gamma$ in the VB-RGG model.

In DC-C-WSNs, a sensor $v$ will share at least one slot with node $u$ if $v$ chooses as its starting point any of $i_{u}-(d-1) \bmod$ $L, \ldots, i_{u}, \ldots i_{u}+(d-1) \bmod L, \ldots, i_{u}-(d-1) \bmod L$ slots. Hence, two sensors have probability $\gamma=\frac{2 d-1}{L}$ of sharing one slot. Now, let the strong $D C-C-W S N$ radius be the radius $r(n)$ that satisfies Theorem 3.3 when $\gamma=\frac{2 d-1}{L}$. We have:

Corollary 4.1. When $\gamma=\frac{2 d-1}{L}<1, P(D C-C$-WSN is connected $) \rightarrow 1$ as $n \rightarrow \infty$ if and only if

$$
r(n)=\sqrt{\frac{\log n+c(n)}{(2 \delta-1 / L) \pi n}},
$$

where $c=\lim _{n \rightarrow \infty} c(n)=\infty$. 


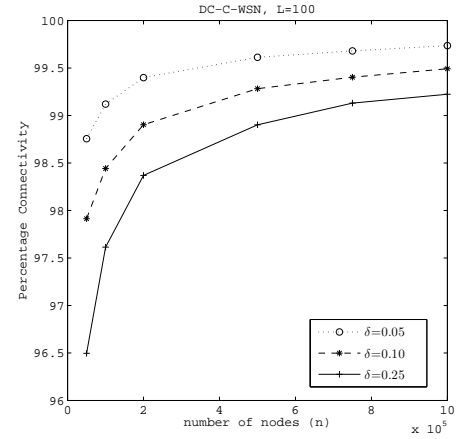

(a)

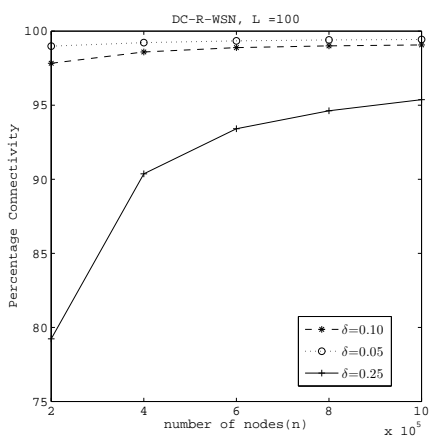

(b)

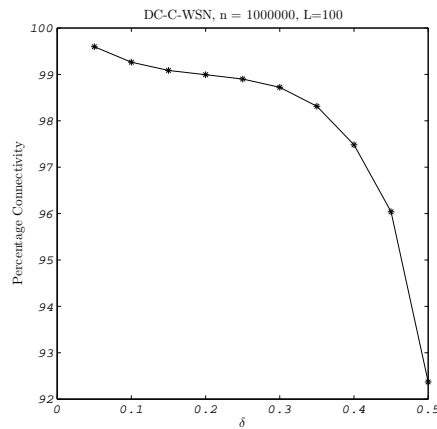

(c)

Figure 1: Strong radius:(a) in DC-C-WSN when $n$ varies (b) in DC-R-WSN when $n$ varies (c) in DC-C-WSN when $\delta$ varies

Note that if $\gamma=\frac{2 d-1}{L}>1$, the radius in (4) goes below the lowest possible radius and it is no longer meaningful. However, $\gamma=\frac{2 d-1}{L}>1$ implies $\delta>1 / 2$, and by Fact 2.1 the RGG radius given in [3] guarantees connectivity.

In DC-R-WSNs, when a node $u$ has chosen $d$ slots, another node $v$ has $d$ possibilities to choose one slot in common with $u$ and the probability of doing that is at least $\delta$ each time. Hence, the probability that two sensors share one slot is $\gamma>\left(1-(1-\delta)^{d}\right)$. Therefore, by Theorem 3.3, the strong $D C-R$-WSN radius must satisfy:

Corollary 4.2. $P(D C-R-W S N$ is connected $) \rightarrow 1$ as $n \rightarrow \infty$ if and only if

$$
r(n)=\sqrt{\frac{\log n+c(n)}{\left(1-(1-\delta)^{d}\right) \pi n}},
$$

where $c=\lim _{n \rightarrow \infty} c(n)=\infty$.

Under the strong radius, Figures $1 \mathrm{a}$ and $1 \mathrm{~b}$ show the percentage of sensors belonging to the largest component when $L=100, c(n)=\log \log n, 10^{5} \leq n \leq 10^{6}, \delta=0.05,0.15$ and 0.25 . For both schemes, fixing a value of $\delta$, the size of the largest connected component increases when $n$ increases since (3) holds for $n \rightarrow+\infty$. On the other hand, fixing a value of $n$, when $\delta$ increases, the the number of nodes in the largest connected component slightly decreases according to the fact that the radius $r(n)$ decreases. Nonetheless, for all the experiments on DC-C-WSNs, more than $90 \%$ of the sensors belong to the largest component. Such a percentage is disregarded by the DC-R-WSNs only for small values of $n$ and large values of $\delta$, as for $n=2 \cdot 10^{5}$ or $n=4 \cdot 10^{5}$ and $\delta=0.25$ (see Figure $1 \mathrm{~b}$ ). This shows that the reliance on the asymptotic condition for $n$ is stronger for DC-R-WSNs than for DC-C-WSNs.

Figures 1c and 2 depict the percentage of sensors belonging to the largest component in both schemes when $n=10^{6}$, $L=100, c(n)=\log \log n$ and $0.1 \leq \delta \leq 0.5$. The results are almost as good as the ones for the weak radius, but the strong radius is much smaller than the weak one (see Table 1). Fig. 2 shows a drop off in the percentage of connectivity when $\delta=0.20$, although the connectivity remains high at more than $90 \%$. The gain between the strong and the weak radius in Table 1 is about a factor of $\sqrt{2}$ for the contiguous scheme, while is more important for the random scheme. In conclusion, the strong connectivity condition un-

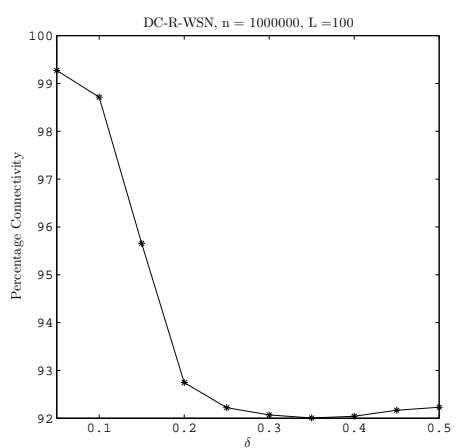

Figure 2: Strong radius in DC-R-WSN when $\delta$ varies.

\begin{tabular}{|l|c|c|c|c|}
\hline & \multicolumn{2}{|c|}{$L=200$} & \multicolumn{2}{c|}{$L=100$} \\
\hline$\delta$ & DC-C-WSN & DC-R-WSN & DC-C-WSN & DC-R-WSN \\
\hline 0.10 & 1.396 & 3.374 & 1.378 & 3.918 \\
\hline 0.15 & 1.402 & 2.591 & 1.390 & 2.702 \\
\hline 0.20 & 1.405 & 2.236 & 1.396 & 2.249 \\
\hline 0.50 & 1.410 & 1.414 & 1.407 & 1.414 \\
\hline
\end{tabular}

Table 1: The ratio of the weak to the strong radius in DC-C-WSN and DC-R-WSN for different $\delta$ and $L$.

doubtedly leads to a great gain in the radius length, and a great energy saving in power transmission for both schemes.

\section{REFERENCES}

[1] A. Bagchi, S. Galhotra, T. Mangla, and C. M. Pinotti. Connectivity in duty-cycled wireless sensor networks. Technical report, Department of Computer Science and Mathematics, University of Perugia, May 2013.

[2] S. K. Das, A. Di Saverio, G. Ghidini, A. Navarra, and C. M. Pinotti. Broadcast analysis in dense duty-cycle sensor networks. In Proc. ICUIMC '12, 2012.

[3] P. Gupta and P. R. Kumar. Critical power for asymptotic connectivity. In Proc. 37th IEEE Conf. on Decision and Control, pages 1106-1110, 1998.

[4] N. A. Pantazis and D. D. Vergados. A survey on power control issues in wireless sensor networks. IEEE Communications Surveys and Tutorials, 9(1-4):86-107, 2007.

[5] M. D. Penrose. On a continuum percolation model. Adv. Appl. Probab., 23(3):546-556, September 1991. 